\title{
Micronutrient Status of Soils from Mango Orchards of YSR Kadapa District Andhra Pradesh and their Relationship with Soil Properties
}

\author{
M. Balakrishna*, T. Giridhara Krishna, K. V. Nagamadhuri, \\ M. V. S. Naidu, P. Sudhakar, B. Ravindra Reddy and K. M. Yuwaraj \\ Department of Soil Science and Agricultural Chemistry, College of Horticulture, \\ Anantharjupeta, Rly.Kodur, Andhra Pradesh, India \\ *Corresponding author
}

\begin{abstract}
A B S T R A C T
Keywords

YSR kadapa district, Mango (Baneeshan), DTPA extractable micronutrients (Fe, $\mathrm{Mn}, \mathrm{Zn}$ and $\mathrm{Cu}$ ) status, Correlation

Article Info

Accepted:

18 November 2020

Available Online:

10 December 2020

To study the DTPA extractable micronutrient status of soil from mango orchards of YSR Kadapa district in all 240 surface and 66 profile samples were collected. The status of DTPA extractable ( $\mathrm{Fe}, \mathrm{Mn}, \mathrm{Zn}$ and $\mathrm{Cu}$ ) micronutrients found to be adequate in most of the mango orchards selected for the present investigation. However, few of the samples were found to be deficient in available $\mathrm{Fe}$ and $\mathrm{Zn}$ content. The correlation study of soil properties with available micronutrients showed that availability of micronutrients in soil get influenced by mechanical composition, maximum water holding capacity, $\mathrm{pH}$, EC, organic carbon, available nitrogen, available phosphorous, available potassium and exchangeable calcium. It was concluded that balanced fertilizer application through integrated nutrient management can sustain fertility status especially in relation to zinc $(\mathrm{Zn})$ and Iron $(\mathrm{Fe})$.
\end{abstract}

\section{Introduction}

Mango (Mangifera indica L.) belonging to family Anacardiaceae is the most important commercially grown fruit crop of Indian subcontinent and particularly in Andhra pradesh. It is one of the most popular, nutritionally rich fruit with unique flavour, fragrance, taste often called "The king of the fruits". The Indian famous and the prime variety of mango, the Baneeshan is chiefly produced in the YSR Kadapa district. The domain of the present research work is YSR Kadapa district is a part of Southern tract and identified as horticulture district of Andhra Pradsh. The district tropical climate with low rainfall (Average annual rainfall of $763 \mathrm{~mm}$, latitude of 14.280 to $14.666 \mathrm{~N}$ and longitude 78.490 to $78.816 \mathrm{E}$. Here, mango is grown on plain to slightly hilly areas under rainfed conditions.

Since, micronutrients present in soil play important role in fruit yield, tree 
development, fruit quality, flowering and fruit size of mango and since deficiency of the micronutrients may cause spongy tissue or delaying maturity of fruits in mango, Balanced application of fertilizers with $\mathrm{Zn}$ and $B$ ensures optimum nutrient concentrations in leaves, which may lead to better quality and a sustainable increase in mango production. South Asian orchard soils are $\mathrm{Zn}$ - or Fe-deficient and may lead to reduced uptake of $\mathrm{N}$ and $\mathrm{K}$ by plants.(Alloy .B ,2009). Low micronutrient use of $\mathrm{Zn}$ and $\mathrm{Fe}$ and poor management practices are mainly responsible for the reduction in yield and fruit quality in mango orchards (Richards. E.L, 2009). The study of micronutrient status of mango orchards of YSR Kadapa district achieves a great consequence.

\section{Materials and Methods}

Twelve mandals encompassing YSR Kadapa district were selected namely I.Chitvel, II. Chinnamandem, III. Chakrayapeta, IV.Galiveedu, V. Kodur, VI. Lakkireddypalle, VII. Penagaluru, VIII. Ramapuram, IX. Rayachoty, X. Sambepalle, XI. T.Sundupalle and XII. Veeraballe. At each Mandal ten Villages of mango growing orchards were selected. From each of the mentioned mango orchards, 20 surface samples $(0$ to $30 \mathrm{~cm})$ and 1-3 profile samples were collected. Thus, in all 240 surface soil samples and 66 profile samples were collected in the month of April 2019. Thus, in all 240 surface soil samples and 66 profile samples were collected in the month of April. The collected soil samples were processed and analysed for DTPA extractable micronutrients (Fe, $\mathrm{Mn}, \mathrm{Zn}$ and $\mathrm{Cu}$ ) as per standard methods (Lindsay and Norvell, 1978). The data obtained from analysis was processed statistically by SAS 9.3, ICAR- 11601386, for studying correlation of soil properties with available micronutrients. The collection of samples, their processing, analysis and statistical analysis of data were done by following standard procedures.

\section{Results and Discussion}

The perusal of data presented in table 1 revealed that the available iron $(\mathrm{Fe})$ status of surface soil samples of all mandals varied from 2.36 to 8.37 with a mean value of 5.36 mg kg-1. In profile samples the available Fe showed a range of 1.87 to 12.13 with an average value of $7.00 \mathrm{mg} \mathrm{kg}-1$. Available Fe status in similar range was also reported by Sankpal (2008) for lateritic soils of Konkan.

In general, all most all mango orchards were found adequate with available Fe with some exceptions showed deficit range in Chakrayapeta, Galiveedu, T. Sundupalle and Veeraballe mandals as per the ratings given by Gajbhiye (1985). The sufficient amount of available $\mathrm{Fe}$ in surface and profiles of soils may be due to laterization processes in which sesquioxides accumulate to increase the $\mathrm{Fe}$ content and also it may be attributed to low $\mathrm{pH}$ and higher organic matter content of mango growing soil (Diwan, 1982).

The data presented in table 2 on overall mean for available manganese (Mn) of different mandals when studied revealed that the available Mn of surface soil samples of all mandals ranged between 10.99 and 17.45 with a mean value of $14.22 \mathrm{mg} \mathrm{kg}-1$. In case of profile soil samples the available $\mathrm{Mn}$ of all mandals varied from 4.54 to 18.68 with an average value of $11.61 \mathrm{mg} \mathrm{kg}-1$.

Similar range for available manganese was also reported by Patil and Meisheri (2004). In general, almost in all the mango orchards available Mn showed a decreasing trend with soil depth. However, all the mango orchards showed sufficient amount of available Mn content on the basis of critical limits given by Gajbhiye (1985). 
Table.1 DTPA-extractable micronutrient (Fe) status of mango orchards ( $\mathrm{mg} \mathrm{kg}^{-1}$ )

\begin{tabular}{|c|c|c|c|c|c|c|c|c|c|c|c|c|}
\hline \multirow{2}{*}{$\begin{array}{l}\text { Mango } \\
\text { orchard } \\
\text { soil depth } \\
\text { (cm) }\end{array}$} & \multicolumn{12}{|c|}{ DTPA-extractable Fe } \\
\hline & I & II & III & IV & $\mathrm{V}$ & VI & VII & VIII & IX & $X$ & $\mathrm{XI}$ & XII \\
\hline \multirow[t]{20}{*}{0 -30 } & 3.24 & 8.06 & 4.73 & 2.19 & 9.68 & 5.33 & 4.6 & 3.55 & 3.23 & 2.96 & 2.67 & 2.42 \\
\hline & 3.21 & 9.12 & 7.19 & 1.45 & 5.28 & 4.41 & 4.55 & 6.01 & 2.48 & 1.55 & 1.27 & 3.08 \\
\hline & 3.39 & 9.45 & 6.88 & 5.64 & 3.68 & 4.68 & 4.5 & 5.04 & 3.09 & 3.18 & 2.41 & 2.04 \\
\hline & 3.73 & 2.83 & 9.69 & 3.32 & 4.97 & 3.95 & 4.46 & 6.44 & 3.67 & 2.88 & 1.19 & 4.25 \\
\hline & 3.82 & 3.75 & 5.04 & 4.39 & 7.11 & 4.34 & 4.34 & 3.28 & 2.29 & 3.96 & 3.76 & 4.22 \\
\hline & 3.98 & 4.02 & 6.32 & 5.55 & 8.65 & 4.97 & 4.53 & 4.29 & 3.68 & 2.97 & 1.88 & 3.64 \\
\hline & 6.92 & 3.79 & 11.45 & 2.37 & 9.68 & 7.27 & 3.46 & 6.21 & 4.85 & 5.02 & 4.67 & 5.12 \\
\hline & 5.12 & 2.94 & 4.86 & 6.66 & 6.58 & 2.8 & 3.49 & 5.59 & 4.12 & 4.24 & 1.98 & 2.74 \\
\hline & 8.45 & 1.98 & 5.57 & 3.37 & 6.78 & 2.65 & 4.02 & 5.55 & 3.94 & 5.14 & 1.83 & 2.45 \\
\hline & 3.65 & 2.75 & 5.34 & 5.54 & 6.42 & 2.98 & 4.11 & 2.79 & 2.95 & 2.34 & 4.34 & 1.11 \\
\hline & 3.42 & 3.85 & 6.67 & 3.75 & 10.98 & 3.56 & 4.15 & 3.74 & 3.18 & 3.15 & 2.21 & 3.63 \\
\hline & 4.05 & 4.15 & 4.45 & 2.18 & 12.38 & 3.04 & 5.62 & 6.15 & 2.89 & 2.57 & 2.18 & 2.16 \\
\hline & 4.12 & 3.25 & 5.26 & 4.78 & 11.12 & 4.95 & 5.54 & 7.12 & 3.17 & 4.02 & 3.44 & 1.84 \\
\hline & 4.19 & 3.85 & 2.85 & 2.22 & 8.31 & 4.55 & 5.57 & 7.66 & 4.2 & 5.78 & 1.05 & 3.15 \\
\hline & 2.82 & 5.62 & 2.98 & 6.15 & 9.24 & 4.42 & 2.36 & 3.55 & 3.01 & 1.38 & 1.97 & 2.74 \\
\hline & 2.95 & 6.15 & 4.95 & 3.18 & 8.27 & 3.48 & 1.99 & 4.56 & 4.62 & 3.32 & 4.28 & 3.74 \\
\hline & 2.76 & 3.55 & 5.32 & 5.09 & 4.48 & 5.56 & 5.83 & 3.66 & 5.05 & 3.56 & 2.42 & 3.57 \\
\hline & 4.2 & 1.89 & 6.48 & 3.61 & 5.06 & 6.97 & 5.78 & 7.12 & 4.45 & 3.76 & 2.43 & 1.53 \\
\hline & 4.21 & 2.25 & 14.34 & 1.99 & 4.56 & 4.32 & 3.74 & 3.12 & 2.84 & 4.84 & 3.08 & 2.77 \\
\hline & 3.46 & 6.05 & 2.75 & 3.25 & 9.68 & 3.78 & 3.64 & 9.26 & 3.05 & 4.23 & 2.42 & 3.05 \\
\hline Mean & 4.08 & 2.36 & 5.60 & 3.81 & 8.07 & 4.42 & 4.23 & 5.88 & 4.08 & 4.02 & 2.80 & 2.64 \\
\hline
\end{tabular}


Table.2 DTPA-extractable micronutrient ( $\mathrm{Zn})$ status of mango orchards ( $\mathrm{mg} \mathrm{kg}^{-1}$ )

\begin{tabular}{|c|c|c|c|c|c|c|c|c|c|c|c|c|}
\hline \multirow{2}{*}{$\begin{array}{l}\text { Mango } \\
\text { orchard } \\
\text { soil depth } \\
\text { (cm) }\end{array}$} & \multicolumn{12}{|c|}{ DTPA-extractable Zn } \\
\hline & I & II & III & IV & $\mathrm{V}$ & VI & VII & VIII & IX & $\mathrm{X}$ & XI & XII \\
\hline \multirow[t]{20}{*}{0 -30 } & 0.67 & 0.42 & 1.39 & 0.79 & 1.19 & 1.56 & 1.91 & 0.86 & 0.91 & 0.53 & 0.47 & 1.03 \\
\hline & 0.52 & 0.36 & 1.72 & 0.59 & 1.56 & 1.49 & 1.78 & 0.65 & 0.46 & 0.49 & 0.46 & 1.92 \\
\hline & 0.68 & 0.62 & 1.31 & 1.21 & 0.84 & 1.61 & 1.92 & 0.46 & 0.58 & 0.52 & 0.44 & 0.55 \\
\hline & 0.28 & 0.95 & 1.13 & 0.46 & 1.46 & $1 . .45$ & 1.38 & 0.55 & 0.74 & 1.09 & 0.37 & 0.75 \\
\hline & 0.51 & 1.11 & 1.19 & 0.66 & 2.49 & 2.49 & 1.57 & 0.59 & 0.56 & 1.01 & 1.19 & 0.72 \\
\hline & 0.31 & 1.01 & 1.23 & 1.02 & 2.56 & 2.67 & 1.67 & 0.41 & 0.65 & 0.76 & 0.21 & 0.37 \\
\hline & 0.25 & 1.15 & 1.33 & 0.56 & 0.52 & 2.56 & 3.01 & 0.46 & 0.42 & 0.57 & 0.15 & 1.04 \\
\hline & 0.36 & 0.66 & 1.49 & 1.68 & 0.49 & 2.16 & 3.21 & 0.66 & 0.34 & 0.58 & 0.1 & 0.58 \\
\hline & 0.47 & 0.58 & 1.32 & 0.74 & 1.35 & 2.19 & 1.98 & 0.55 & 1.21 & 1.15 & 0.27 & 1.42 \\
\hline & 0.77 & 0.72 & 1.21 & 1.11 & 1.32 & 1.45 & 2.03 & 0.36 & 0.78 & 0.41 & 0.32 & 0.4 \\
\hline & 0.26 & 0.49 & 0.99 & 0.89 & 1.1 & 1.19 & 2.01 & 0.86 & 0.49 & 0.61 & 0.04 & 0.8 \\
\hline & 0.73 & 0.56 & 1.29 & 0.57 & 1.05 & 1.57 & 2.38 & 0.88 & 0.91 & 0.52 & 0.08 & 1.14 \\
\hline & 1.02 & 0.47 & 1.07 & 1.35 & 0.53 & 2.22 & 2.24 & 0.81 & 0.59 & 0.94 & 0.19 & 1.76 \\
\hline & 0.89 & 0.33 & 1.26 & 0.61 & 0.93 & 2.23 & 2.36 & 0.71 & 0.49 & 0.89 & 0.26 & 0.97 \\
\hline & 1.12 & 0.72 & 1.15 & 1.38 & 1.15 & 1.87 & 1.52 & 0.49 & 1.23 & 1.01 & 0.14 & 0.97 \\
\hline & 0.54 & 0.54 & 2.01 & 0.84 & 0.47 & 1.57 & 1.54 & 0.55 & 0.72 & 0.43 & 0.29 & 0.79 \\
\hline & 1.11 & 0.39 & 1.41 & 0.68 & 1.14 & 1.23 & 3.04 & 0.46 & 0.54 & 0.56 & 0.19 & 1.42 \\
\hline & 1.17 & 0.28 & 1.57 & 0.42 & 1.26 & 1.6 & 3.87 & 0.64 & 0.39 & 0.39 & 0.3 & 1.4 \\
\hline & 0.63 & 0.41 & 2.49 & 0.63 & 0.55 & 1.41 & 1.01 & 0.25 & 0.28 & 1.04 & 0.18 & 1.24 \\
\hline & 0.47 & 0.59 & 1.19 & 0.44 & 1.19 & 1.05 & 1.26 & 0.45 & 0.41 & 0.88 & 0.26 & 1.62 \\
\hline Mean & 0.88 & 0.42 & 1.56 & 0.73 & 0.77 & 1.60 & 2.20 & 0.51 & 0.55 & 0.76 & 0.11 & 1.29 \\
\hline
\end{tabular}


Table.3 DTPA-extractable micronutrient $(\mathrm{Cu})$ status of mango orchards $\left(\mathrm{mg} \mathrm{kg}^{-1}\right)$

\begin{tabular}{|c|c|c|c|c|c|c|c|c|c|c|c|c|}
\hline \multirow{2}{*}{$\begin{array}{l}\text { Mango } \\
\text { orchard } \\
\text { soil depth }(\mathrm{cm})\end{array}$} & \multicolumn{12}{|c|}{ DTPA-extractable Cu } \\
\hline & I & II & III & IV & V & VI & VII & VIII & IX & $\mathrm{X}$ & XI & XII \\
\hline \multirow[t]{20}{*}{$0-30$} & 1.65 & 1.15 & 2.2 & 0.55 & 2.12 & 1.5 & 2.05 & 1.95 & 1.54 & 0.95 & 1.2 & 1.03 \\
\hline & 1.42 & 0.76 & 2.54 & 0.39 & 2.26 & 0.96 & 2.24 & 1.86 & 1.44 & 1.06 & 0.83 & 1.92 \\
\hline & 1.55 & 0.61 & 2.58 & 2.14 & 2.36 & 1.76 & 2.16 & 1.75 & 1.67 & 0.55 & 0.68 & 0.55 \\
\hline & 1.48 & 1.56 & 2.17 & 2.79 & 2.33 & 1.71 & 2.6 & 1.66 & 1.54 & 2.01 & 0.52 & 0.75 \\
\hline & 1.36 & 0.35 & 1.62 & 1.37 & 3.05 & 2.97 & 2.56 & 1.25 & 1.43 & 0.94 & 1.4 & 0.72 \\
\hline & 1.58 & 2.13 & 1.08 & 2.31 & 3.24 & 1.11 & 2.72 & 1.88 & 2.36 & 1.17 & 1.04 & 0.37 \\
\hline & 2.57 & 0.95 & 2.49 & 1.09 & 3.11 & 2.85 & 1.66 & 1.14 & 0.71 & 0.97 & 1.08 & 1.04 \\
\hline & 2.14 & 0.92 & 1.54 & 1.75 & 2.05 & 1.92 & 1.57 & 1.14 & 0.82 & 1.76 & 1.54 & 0.58 \\
\hline & 2.62 & 1.03 & 1.66 & 1.29 & 2.11 & 2.11 & 1.91 & 1.64 & 0.92 & 0.82 & 1.08 & 1.42 \\
\hline & 1.38 & 1.42 & 2.31 & 2.01 & 2.1 & 1.38 & 1.87 & 1.55 & 1.53 & 1.06 & 1.64 & 0.4 \\
\hline & 1.21 & 1.06 & 1.39 & 1.56 & 1.71 & 1.46 & 2.21 & 1.62 & 1.4 & 1.54 & 1.23 & 0.8 \\
\hline & 1.88 & 0.66 & 1.5 & 0.98 & 1.76 & 1.41 & 1.94 & 1.58 & 1.46 & 0.79 & 1.41 & 1.14 \\
\hline & 1.98 & 1.62 & 1.15 & 2.21 & 1.71 & 1.5 & 1.81 & 1.54 & 1.66 & 0.97 & 0.98 & 1.76 \\
\hline & 1.96 & 0.54 & 1.12 & 2.12 & 2.47 & 1.63 & 1.89 & 2.24 & 1.62 & 0.15 & 1.64 & 0.97 \\
\hline & 1.81 & 0.95 & 1.38 & 2.46 & 2.68 & 1.39 & 1.45 & 1.12 & 1.54 & 2.02 & 1.85 & 0.97 \\
\hline & 1.66 & 1.15 & 1.58 & 0.87 & 2.52 & 1.8 & 1.15 & 1.53 & 0.93 & 0.58 & 2.62 & 0.79 \\
\hline & 1.81 & 0.78 & 1.59 & 2.21 & 1.23 & 1.58 & 2.67 & 2.36 & 1.01 & 0.66 & 1.76 & 1.42 \\
\hline & 2.03 & 0.34 & 1.8 & 1.46 & 1.22 & 1.91 & 2.76 & 2.45 & 2.78 & 0.56 & 1.44 & 1.4 \\
\hline & 2.16 & 1.09 & 2.97 & 0.54 & 1.33 & 2.59 & 1.37 & 1.19 & 2.04 & 1.41 & 1.37 & 1.03 \\
\hline & 1.87 & 0.64 & 2.11 & 1.12 & 2.12 & 1.85 & 1.56 & 1.46 & 1.79 & 1.35 & 1.66 & 1.92 \\
\hline Mean & 2.01 & 0.82 & 1.64 & 1.55 & 1.65 & 1.86 & 1.70 & 1.68 & 1.69 & 0.99 & 1.88 & 1.32 \\
\hline
\end{tabular}


Table.4a DTPA-extractable micronutrient (Mn) status of mango orchards ( $\mathrm{mg} \mathrm{kg}^{-1}$ )

\begin{tabular}{|c|c|c|c|c|c|c|c|c|c|c|c|c|}
\hline \multirow{2}{*}{$\begin{array}{l}\text { Mango } \\
\text { orchard } \\
\text { soil depth }(\mathrm{cm})\end{array}$} & \multicolumn{12}{|c|}{ DTPA-extractable Mn } \\
\hline & I & II & III & IV & V & VI & VII & VIII & IX & $\mathrm{X}$ & XI & XII \\
\hline \multirow[t]{20}{*}{$0-30$} & 27.9 & 16.07 & 10.32 & 8.79 & 13.66 & 27.93 & 12.41 & 17.45 & 18.33 & 6.62 & 17.13 & 12.66 \\
\hline & 25.64 & 12.89 & 39.05 & 11.25 & 7.53 & 26.57 & 13.65 & 16.36 & 19.56 & 6.72 & 12.21 & 13.25 \\
\hline & 27.67 & 16.45 & 25.23 & 9.45 & 7.68 & 27.14 & 12.68 & 18.44 & 17.17 & 6.79 & 13.62 & 10.87 \\
\hline & 33.33 & 17.42 & 28.13 & 6.69 & 7.69 & 25.51 & 38.16 & 19.47 & 9.29 & 8.99 & 17.94 & 21.78 \\
\hline & 31.17 & 18.21 & 18.15 & 13.54 & 8.48 & 50.64 & 35.67 & 15.46 & 10.17 & 9.73 & 15.28 & 26.15 \\
\hline & 33.54 & 17.05 & 12.98 & 8.82 & 10.55 & 52.47 & 39.41 & 14.55 & 10.36 & 10.38 & 12.86 & 15.59 \\
\hline & 11.93 & 28.05 & 7.97 & 12.15 & 16.56 & 50.55 & 24.02 & 15.22 & 17.28 & 13.71 & 14.15 & 20.96 \\
\hline & 12.54 & 15.55 & 21.92 & 26.68 & 37.02 & 11.3 & 25.62 & 15.66 & 18.01 & 14.13 & 22.02 & 15.64 \\
\hline & 11.67 & 18.4 & 20.11 & 20.1 & 37.72 & 12.56 & 16.5 & 16.33 & 17.56 & 13.96 & 32.1 & 11.54 \\
\hline & 6.49 & 25.75 & 29.54 & 15.56 & 37.9 & 15.6 & 16.77 & 12.33 & 21.45 & 38.31 & 12.38 & 13.24 \\
\hline & 6.98 & 17.65 & 30.3 & 9.87 & 9.48 & 16.12 & 16.05 & 18.66 & 20.79 & 37.71 & 16.3 & 11.28 \\
\hline & 8.1 & 8.55 & 26.57 & 12.12 & 10.41 & 15.58 & 19.88 & 19.12 & 19.85 & 37.15 & 11.64 & 22.74 \\
\hline & 8.47 & 9.65 & 38.13 & 10.25 & 9.96 & 23.64 & 18.58 & 12.25 & 6.31 & 17.72 & 12.28 & 11.44 \\
\hline & 8.38 & 7.14 & 11.83 & 17.56 & 11.56 & $24 . .23$ & 21.07 & 10.23 & 6.69 & 18.37 & 13.47 & 19.15 \\
\hline & 25.77 & 18.15 & 15.68 & 15.54 & 11.29 & 20.15 & 20.68 & 10.25 & 7.14 & 17.67 & 1874 & 15.28 \\
\hline & 25.06 & 20.25 & 23.64 & 7.85 & 11.3 & 17.18 & 22.68 & 8.36 & 21.05 & 11.63 & 11.35 & 12.96 \\
\hline & 25.62 & 17.75 & 19.19 & 15.04 & 7.95 & 19.86 & 17.59 & 9.56 & 24.27 & 12.23 & 21.42 & 21.15 \\
\hline & 16.94 & 13.5 & 17.18 & 10.15 & 7.74 & 17.29 & 17.89 & 11.56 & 21.32 & 11.08 & 11.43 & 15.16 \\
\hline & 16.57 & 19.45 & 12.64 & 13.25 & 13.66 & 19.19 & 7.06 & 12.53 & 15.74 & 5.04 & 20.54 & 16.11 \\
\hline & 18.97 & 18.25 & 11.56 & 8.54 & 7.53 & 20.28 & 7.02 & 17.45 & 15.12 & 5.51 & 17.13 & 12.04 \\
\hline Mean & 12.99 & 16.27 & 17.45 & 13.23 & 12.30 & 16.89 & 14.15 & 10.99 & 16.62 & 17.24 & 17.13 & 15.50 \\
\hline
\end{tabular}


Table.4b DTPA-extractable micronutrients status of mango orchards Soil profiles at different depths $\left(\mathrm{mg} \mathrm{kg}^{-1}\right)$

\begin{tabular}{|c|c|c|c|c|}
\hline 1.Chitvel Profile depth (meters) & $\mathbf{F e}$ & $\mathbf{Z n}$ & $\mathbf{C u}$ & Mn \\
\hline $0.00-0.18$ & 4.14 & 1.73 & 1.88 & 18.10 \\
\hline 0.18-0.40 & 3.49 & 1.41 & 1.56 & 17.78 \\
\hline $0.40-0.55$ & 2.28 & 1.21 & 0.99 & 15.56 \\
\hline Mean & 3.32 & 1.45 & 1.47 & $\mathbf{1 7 . 1 4}$ \\
\hline \multicolumn{5}{|l|}{ 2.Chakrayapeta } \\
\hline $0.00-0.30$ & 9.30 & 0.56 & 1.27 & 15.52 \\
\hline $0.30-0.74$ & 10.24 & 0.53 & 1.12 & 13.52 \\
\hline $0.74-1.23$ & 8.29 & 0.43 & 0.89 & 13.78 \\
\hline $1.23-1.75$ & 5.56 & 0.36 & 0.60 & 12.53 \\
\hline $1.75-2.00$ & 7.40 & 0.29 & 0.31 & 10.25 \\
\hline Mean & 8.158 & 0.43 & 0.83 & 13.12 \\
\hline \multicolumn{5}{|l|}{ 3.Chinnamandem } \\
\hline $0.00-0.30$ & 3.34 & 0.47 & 0.82 & 10.54 \\
\hline $0.30-0.62$ & 1.49 & 0.44 & 0.56 & 8.46 \\
\hline $0.62-0.76$ & 1.81 & 0.38 & 0.34 & 8.25 \\
\hline $0.76-1.00$ & 0.85 & 0.29 & 0.28 & 6.50 \\
\hline Mean & 1.87 & 0.39 & 0.50 & 8.43 \\
\hline \multicolumn{5}{|l|}{ 4.Galiveedu I } \\
\hline $0.00-0.30$ & 3.77 & 1.21 & 2.53 & 19.41 \\
\hline $0.30-0.60$ & 3.21 & 0.98 & 2.22 & 18.25 \\
\hline $0.60-0.93$ & 2.69 & 0.72 & 2.14 & 18.05 \\
\hline $0.93-1.23$ & 2.84 & 0.72 & 1.59 & 17.01 \\
\hline $1.23-1.52$ & 2.02 & 0.50 & 1.36 & 16.98 \\
\hline Mean & 2.90 & 0.82 & 1.96 & 17.94 \\
\hline \multicolumn{5}{|l|}{ 5.Galiveedu II } \\
\hline $0.00-0.20$ & 4.17 & 1.15 & 2.63 & 23.58 \\
\hline $0.20-0.76$ & 4.05 & 1.24 & 2.54 & 20.50 \\
\hline 0.76-0.99 & 3.09 & 1.80 & 2.33 & 17.28 \\
\hline $0.99-1.20$ & 2.31 & 1.91 & 2.49 & 15.09 \\
\hline Mean & 3.40 & 1.52 & 2.49 & 19.11 \\
\hline Profile Mean & 2.38 & 1.17 & 2.22 & 18.52 \\
\hline \multicolumn{5}{|l|}{ 6.Kodur I } \\
\hline 0.00-0.17 & 3.82 & 0.55 & 2.98 & 6.53 \\
\hline 0.17-0.49 & 3.45 & 0.38 & 1.97 & 5.14 \\
\hline 0.49-0.79 & 2.18 & 0.38 & 1.66 & 4.40 \\
\hline $0.79-1.21$ & 2.05 & 0.27 & 1.52 & 3.54 \\
\hline $1.21-1.70$ & 1.19 & 0.16 & 1.41 & 3.12 \\
\hline Mean & 2.53 & 0.34 & 1.90 & 4.54 \\
\hline \multicolumn{5}{|l|}{ 7.Kodur II } \\
\hline $0.00-0.20$ & 10.98 & 1.10 & 1.71 & 9.48 \\
\hline $0.20-0.41$ & 8.81 & 0.87 & 1.40 & 7.46 \\
\hline $0.41-0.70$ & 7.11 & 0.62 & 1.26 & 5.55 \\
\hline $0.70-0.90$ & 5.17 & 0.43 & 0.99 & 3.78 \\
\hline Mean & 8.01 & 0.75 & 1.34 & 6.56 \\
\hline \multicolumn{5}{|l|}{ 8.Kodur III } \\
\hline $0.00-0.21$ & 11.98 & 2.49 & 3.05 & 9.48 \\
\hline $0.21-0.51$ & 8.85 & 1.88 & 2.78 & 7.24 \\
\hline 0.51-0.81 & 5.56 & 1.71 & 1.85 & 6.67 \\
\hline 0.81-1.10 & 5.01 & 1.19 & 1.66 & 3.39 \\
\hline
\end{tabular}




\begin{tabular}{|c|c|c|c|c|}
\hline Mean & 7.85 & 1.81 & 2.33 & 6.69 \\
\hline Profile Mean & 6.13 & 0.96 & 1.85 & 5.93 \\
\hline \multicolumn{5}{|c|}{ 9.Lakkireddypalle } \\
\hline $0.00-0.25$ & 6.20 & 1.23 & 2.01 & 15.21 \\
\hline $0.25-0.60$ & 5.52 & 1.17 & 2.59 & 14.40 \\
\hline $0.60-0.55$ & 4.30 & 0.89 & 2.62 & 16.08 \\
\hline $0.55-1.13$ & 4.01 & 0.83 & 2.58 & 18.29 \\
\hline Mean & 5.00 & 1.03 & 2.45 & 15.99 \\
\hline \multicolumn{5}{|l|}{ 10.Penagaluru } \\
\hline $0.00-0.16$ & 4.33 & 1.25 & 0.42 & 16.57 \\
\hline $0.16-0.40$ & 4.12 & 1.05 & 0.33 & 14.52 \\
\hline $0.40-0.69$ & 3.39 & 0.72 & 0.27 & 11.05 \\
\hline 0.69-0.89 & 2.19 & 0.56 & 0.26 & 8.98 \\
\hline Mean & 3.50 & 0.89 & 0.32 & 12.78 \\
\hline \multicolumn{5}{|l|}{ 11.Rayachoty } \\
\hline $0.00-0.19$ & 3.35 & 0.54 & 0.54 & 8.57 \\
\hline 0.19-0.41 & 2.90 & 0.46 & 0.48 & 8.16 \\
\hline $0.41-0.65$ & 2.12 & 0.37 & 0.44 & 6.29 \\
\hline $0.65-1.00$ & 2.01 & 0.21 & 0.37 & 4.95 \\
\hline Mean & 2.59 & 0.39 & 0.45 & 6.99 \\
\hline \multicolumn{5}{|c|}{ 12.Ramapuram I } \\
\hline $0.00-0.20$ & 12.54 & 0.65 & 0.42 & 20.16 \\
\hline $0.20-0.49$ & 11.78 & 0.48 & 0.36 & 18.58 \\
\hline $0.49-0.67$ & 11.48 & 0.23 & 0.24 & 17.32 \\
\hline Mean & 11.93 & 0.45 & 0.34 & 18.68 \\
\hline \multicolumn{5}{|c|}{ 13.RamapuramII } \\
\hline $0.00-0.20$ & 14.31 & 0.52 & 0.76 & 14.25 \\
\hline $0.20-0.41$ & 12.52 & 0.24 & 0.42 & 12.36 \\
\hline 0.41-0.70 & 10.19 & 0.11 & 0.24 & 10.79 \\
\hline Mean & 12.34 & 0.29 & 0.47 & 12.46 \\
\hline Prifile Mean & 12.13 & 0.37 & 0.40 & 15.57 \\
\hline \multicolumn{5}{|l|}{ 14.Sambepalle } \\
\hline $0.00-0.30$ & 3.12 & 0.46 & 2.64 & 13.5 \\
\hline $0.30-0.61$ & 3.05 & 0.42 & 1.98 & 10.96 \\
\hline 0.61-0.99 & 2.76 & 0.33 & 1.71 & 7.23 \\
\hline 0.99-1.16 & 1.48 & 0.26 & 1.16 & 7.10 \\
\hline Mean & 2.60 & 0.36 & 1.87 & 9.69 \\
\hline \multicolumn{5}{|c|}{ 15.T.Sundupalle } \\
\hline $0.00-0.18$ & 3.80 & 3.28 & 0.69 & 12.82 \\
\hline 0.18-0.50 & 3.75 & 3.12 & 0.31 & 10.23 \\
\hline $0.50-0.71$ & 2.92 & 2.20 & 0.26 & 7.80 \\
\hline $0.71-1.22$ & 2.56 & 2.10 & 0.22 & 6.58 \\
\hline $1.22-1.50$ & 1.73 & 1.28 & 0.15 & 5.11 \\
\hline Mean & 2.95 & 2.39 & 0.32 & 8.50 \\
\hline \multicolumn{5}{|l|}{ 16.Veeraballe } \\
\hline $0.00-0.20$ & 5.56 & 0.55 & 0.92 & 14.86 \\
\hline $0.20-0.42$ & 4.99 & 0.40 & 0.52 & 12.24 \\
\hline $0.42-0.61$ & 3.42 & 0.25 & 0.49 & 9.36 \\
\hline $0.61-90$ & 1.90 & 0.27 & 0.45 & 7.91 \\
\hline $0.90-1.20$ & 1.35 & 1.02 & 0.39 & 6.32 \\
\hline Mean & 3.44 & 0.49 & 0.55 & 10.13 \\
\hline
\end{tabular}


Table.5 Correlation between available micronutrients and physico-chemical properties of soil

\begin{tabular}{|l|l|l|l|l|}
\hline Soil properties & Available Fe & Available Mn & Available Zn & Available Cu \\
\hline Sand & $0.1909^{*}$ & $0.2715^{*}$ & $0.3282^{*}$ & $0.4557^{*}$ \\
\hline Silt & 0.1078 & 0.0134 & $0.2372^{*}$ & 0.0028 \\
\hline Clay & $-0.2609^{*}$ & $-0.2665^{*}$ & $-0.4156^{*}$ & $-0.3899^{*}$ \\
\hline BD & 0.1220 & 0.0693 & -0.0523 & 0.0365 \\
\hline Pd & 0.0732 & 0.0998 & -0.0312 & -0.0155 \\
\hline MWHC & $-0.2975^{*}$ & $-0.2165^{*}$ & $-0.1837^{*}$ & -0.1311 \\
\hline pH & $-0.2072^{*}$ & $-0.3777^{*}$ & $-0.3006^{*}$ & $-0.1781^{*}$ \\
\hline EC & 0.0908 & -0.0335 & 0.0905 & 0.0579 \\
\hline OC & $0.3921^{*}$ & $0.3106^{*}$ & 0.1607 & 0.0553 \\
\hline $\begin{array}{l}\text { Available } \\
\text { Nitrogen }\end{array}$ & $0.2571^{*}$ & $0.4319^{*}$ & $0.3691^{*}$ & $0.4359^{*}$ \\
\hline Available P2O5 & $0.2290^{*}$ & $0.3017^{*}$ & 0.0481 & 0.0312 \\
\hline Available K2O & $-0.2862^{*}$ & -0.1452 & -0.1110 & 0.0602 \\
\hline $\begin{array}{l}\text { Exchangeable } \\
\text { Ca2+ }\end{array}$ & 0.1094 & $0.3598^{*}$ & $0.2282^{*}$ & $0.4204^{*}$ \\
\hline $\begin{array}{l}\text { Exchangeable } \\
\text { Mg2+ }\end{array}$ & -0.0848 & 0.1003 & 0.0377 & 0.0772 \\
\hline *Significant at 5 per cent level & & & \\
\hline
\end{tabular}

*Significant at 5 per cent level

Data on overall mean for available zinc of different mandals showed that the available $\mathrm{Zn}$ status of surface soil samples of all mandals had a variation between 0.51 and 2.20 with an average value of $1.35 \mathrm{mg} \mathrm{kg}^{-1}$. For profile soil samples the available zinc ranged from 0.34 to 1.45 with a mean value of $0.89 \mathrm{mg} \mathrm{kg}^{-1}$ (Table 3). These results are in conformity with Gaidhani (2008). At all the profiles available $\mathrm{Zn}$ showed a decreasing trend with soil depth. Further, from data it was observed that most of the soil samples of mango orchards had sufficient amount of available zinc. However, few soil samples at Chakrayapeta, Ramapuram, Rayachoty and T.Sudupalle mango orchards were found to be deficient in available zinc content as per the critical limits given by Gajbhiye (1985).

As seen from table $4 a$ and $b$, the data on available copper status of different mandals revealed that the available $\mathrm{Cu}$ of surface soil samples varied from 0.82 to 2.012 with a mean value of $1.41 \mathrm{mg} \mathrm{kg}-1$. For profile soil samples the available copper had a range of 0.32 to 2.45 with an average value of $1.38 \mathrm{mg}$ $\mathrm{kg}^{-1}$. In general, available copper content showed a decreasing trend with soil depth in profile samples. Similar findings were also reported by Suryavanshi (2010). Also, the available $\mathrm{Cu}$ in most of the soil samples of mango orchards was found to be adequate critical limit given by Gajbhiye (1985).

The data from table 5 showed that available $\mathrm{Fe}$ exhibited positive and significant correlation with sand $(r=0.1909)$ whereas negative and significant relationship was found with clay content $(r=-0.2609)$. The maximum water holding capacity was found to be negatively and significantly correlated with available iron content in the soil $(\mathrm{r}=$ 0.2975). In case of $\mathrm{pH}(\mathrm{r}=-0.2072)$ negative but significant correlation with available $\mathrm{Fe}$ was observed. However, organic carbon was positively and significantly correlated with 
available iron content $(\mathrm{r}=-0.267)$. Negative but significant correlation of available Fe may be due to formation of insoluble higher oxides of Fe at higher $\mathrm{pH}$ (Patil and Meisheri, 2004; Patil et al., 2003). The available Fe content established positive significant relationship with available nitrogen $(\mathrm{r}=0.257)$ and available phosphorus $(\mathrm{r}=0.2290)$ whereas with available $\mathrm{K} 2 \mathrm{O}$, a negative but significant relationship was observed $(r=-0.2862)$ with available Fe content.

The sand content had shown positive and significant influence on the availability of $\mathrm{Mn}$ in the soil $(r=0.271)$ while the clay content showed negative and significant correlation with available $\mathrm{Mn}(\mathrm{r}=-0.2665)$. Increase in maximum water holding capacity $(r=-0.2265$ and $\mathrm{pH}(\mathrm{r}=-0.3777)$ were found to have inverse effect on availability of Mn. Further data on correlation also revealed that available $\mathrm{Mn}$ exhibited positive and significant correlation with organic carbon $(\mathrm{r}$ $=0.3106)$, available nitrogen $(\mathrm{r}=0.4319)$, available phosphorus $(\mathrm{r}=0.3017)$ and exchangeable $\mathrm{Ca}^{+2}(\mathrm{r}=0.3598)$. A positive correlation of available $\mathrm{Mn}$ with organic carbon indicated that availability of $\mathrm{Mn}$ increases with increase in organic matter for the soils of Konkan Region (Patil et al., 2003).

Zinc was found to be positively and significantly correlated with sand $(r=0.3282)$ and silt content $(r=0.2372)$ while it showed negative, but significant relationship with clay content $(\mathrm{r}=-0.4156)$. Available $\mathrm{Zn}$ had a positive, but significant correlation $(\mathrm{r}=$ 0.1607) with organic carbon content in soil. Increase in maximum water holding capacity $(\mathrm{r}=-0.1837)$ and $\mathrm{pH}(\mathrm{r}=-0.3006)$ resulted in decrease in available zinc content in soil. Available nitrogen $(\mathrm{r}=0.3691)$ and exchangeable calcium $(r=0.0067)$ were found to be positively and significantly correlated with available $\mathrm{Zn}$ content in soil.
Similar findings were noticed by Mahajan (2001).

Available copper was found to increase significantly with sand content in the soil (r $=0.4557)$, while clay content $(r=-0.3899)$ had negative but significant correlation with available $\mathrm{Cu}$. From table 5, it was also observed available copper content had a negative, but significant correlation with $\mathrm{pH}$ $(\mathrm{r}=-0.1781)$. Available nitrogen $(\mathrm{r}=0.4359)$ and exchangeable calcium $(\mathrm{r}=0.4204)$ was correlated positively and significantly with available $\mathrm{Cu}$ content in soil.

From the data, it could be concluded the status of DTPA extractable (Fe, Mn, $\mathrm{Zn}$ and $\mathrm{Cu}$ ) micronutrients found to be adequate in most of the mango orchards selected for the present investigation. However, 2\%,8\% 17\% samples from Chakrayapeta, Ramapuram and T.Sundupalle locations were respectively found to be deficient in available $\mathrm{Fe}$ and $\mathrm{Zn}$ content. The correlation study indicated that the mechanical composition, maximum water holding capacity, $\mathrm{pH}$, EC, organic carbon, available nitrogen, available phosphorous, available potassium and exchangeable calcium of soil affected the availability of micronutrients. From the results, it is suggested that in future, balanced fertilizer application through integrated nutrient management should be followed to sustain fertility status especially in relation to calcium $(\mathrm{Ca} 2+)$, magnesium $\left(\mathrm{Mg}^{2+}\right)$, zinc $(\mathrm{Zn})$ and copper $(\mathrm{Cu})$.

\section{References}

Alloway, B. Soil factors associated with zinc deficiency in crops and humans. Environ. Geochem. Health 2009,31, 537-548.

Diwan, P. M. (1982). Micronutrient status of the soils from bench terraced area of the Central Farm, Wakawali. M.Sc.(Agri.) 
Thesis submitted to Konkan Krishi Vidyapeeth, Dapoli, Dist. Ratnagiri Maharashtra).

Gaidhani, S. M. (2008). Effect of integrated nutrient management on yield, partitioning and uptake by rice and on fertility status of lateritic soils of Konkan. M.Sc. (Agri.) Thesis submitted to Balasaheb Sawant Konkan Krishi Vidyapeeth, Dapoli, Dist. Ratnagiri, Maharashtra.

Gajbhiye (1985). Common extractants and critical limits for available $\mathrm{Fe}, \mathrm{Mn}, \mathrm{Zn}$ and $\mathrm{Cu}$ in soil. J. Maharashtra Agril.Univ., 10(3) : 239-241.

Lindsay, W. L. and W. A. Norvell (1978). Development of DTPA soil test for Zn, $\mathrm{Fe}, \mathrm{Mn}$ and $\mathrm{Cu}$. Proc. Soil Sci. Soc. Amer., 42: 421-428.

Mahajan, T. S. (2001). Status and distribution of micronutrients in relation to the properties of lateritic soils under mango orchards in South Konkan. M. Sc. (Agri.) Thesis submitted to Balasaheb Sawant Konkan Krishi Vidyapeeth, Dapoli, Dist. Ratnagiri, Maharashtra.

Patil, K. D. and M. B. Meisheri (2004). Minerological studies and DTPAExtractable $\mathrm{Zn}, \mathrm{Cu}, \mathrm{Mn}$ and $\mathrm{Fe}$ in representative soils of Konkan region. $J$. Maharashtra Agril. Univ., 29(1): 4-8.
Patil, K. D., M. B. Meisheri, D. J. Dabke and D. S. Bagade (2003). Distribution of DTPA-extractable cinc, copper, iron and manganese contents in rice soils of Konkan. J. Soils and Crops, 13(1): 8590.

Pereira, A. J., A. S. Chavan and P. A. Varade (1986). Physicochemical properties and micronutrients status of the mango orchard hill soils of Konkan. $J$. Maharashtra Agric. Univ., 11(2): 134136.

Richards, E.L. The Mango-Botany, Production and Uses, 2nd ed.; CABI: Oxford, UK, 2009; p. 680.

Sankpal, A. A. (2008). Studies on physicochemical properties of lateritic soils of Agriculture Research Station, Phondaghat (Sindhudurg). M.Sc. (Agri.) Thesis submitted to Balasaheb Sawant Konkan Krishi Vidyapeeth, Dapoli, Dist. Ratnagiri, Maharashtra.

Suryavanshi, A. V. (2010). Micronutrient status and its relationship with soil properties in mound planted mango orchards of Sindhudurg district (Konkan region, M.S.). M.Sc. (Agri.) Thesis submitted to Balasaheb sawant Konkan Krishi Vidyapeeth, Dapoli, Dist. Ratnagiri, Maharashtra.

\section{How to cite this article:}

Balakrishna, M., T. Giridhara Krishna, K. V. Nagamadhuri, M. V. S. Naidu, P. Sudhakar, B. Ravindra Reddy and Yuwaraj, K. M. 2020. Micronutrient Status of Soils from Mango Orchards of YSR Kadapa District Andhra Pradesh and their Relationship with Soil Properties. Int.J.Curr.Microbiol.App.Sci. 9(12): 2834-2844. doi: https://doi.org/10.20546/ijcmas.2020.912.338 